\title{
Wind Farm Wake-Steering Exploration During Grid Curtailment
}

\author{
A THESIS \\ SUBMITTED TO THE FACULTY OF THE GRADUATE SCHOOL \\ OF THE UNIVERSITY OF MINNESOTA \\ BY
}

Jordan Hoyt

IN PARTIAL FULFILLMENT OF THE REQUIREMENTS FOR THE DEGREE OF MASTER OF SCIENCE

Peter Seiler Ph.D.

August, 2020 
(C) Jordan Hoyt 2020

ALL RIGHTS RESERVED 


\section{Acknowledgements}

This material is based upon work supported by the National Science Foundation Graduate Research Fellowship Program under Grant No. CON-75851, project 00074041. Any opinions, findings, and conclusions or recommendations expressed in this material are those of the author(s) and do not necessarily reflect the views of the National Science Foundation.

This research would not be possible without the hard work by the researchers at the National Wind Technology Center, particularly those that made the FAST.Farm simulation software possible. The research was supported by The Xcel Renewable Development Fund entitled Simulation, Measurement, Modeling, and Control of Wind Plant Power. 


\section{Dedication}

To my grandmother Sally Willis who often texted to remind me to stay warm during the Minnesota winters. My late grandfather caught frostbite on the tip of his ear while serving in Germany and since then she's gone above and beyond the call of duty as a wife, mother, and grandmother to keep her family warm, both thermally and spiritually.

"Energy to Keep Us Free"

- A plaque on the Wind Systems Building, United States Department of Energy, dedicated July 17, 1981 


\begin{abstract}
Wind farm wake steering is an active topic in the wind community. Yaw-induced wake steering in wind farms has shown significant increases in total wind farm power output. Unfortunately, sensor uncertainty and model uncertainty often make pure model-driven approaches less effective. Due to the mechanical and financial downsides associated with experimental wake steering, collecting useful data to verify model-based approaches is often viewed as too risky. However, electric grid curtailment periods offer the opportunity to experiment with minimal mechanical and financial risks. A novel data acquisition process is presented that utilizes grid curtailment periods for wake steering experimentation. This method curtails the total wind farm power output while yaw sweeping the upstream turbine to discover the optimal yaw angle for wake-steering. The optimal yaw angle can then be used in regular uncurtailed periods to increase total power output.
\end{abstract}




\section{Contents}

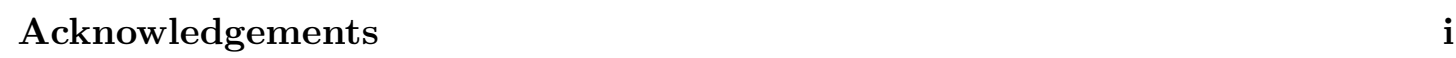

Dedication ii

Abstract iii

List of Tables vi vi v v vi

List of Figures vii

\begin{tabular}{lll}
\hline 1 & Overview & 1
\end{tabular}

\begin{tabular}{|ll|l}
\hline 2 & Intro & 3
\end{tabular}

3 Background 5

3.1 Individual Turbine Theory $\ldots \ldots \ldots \ldots \ldots$

3.2 Wind Farm Theory $\ldots \ldots \ldots \ldots \ldots$. . . . . . . . . . . . . 7

3.3 FAST.Farm Model . . . . . . . . . . . . . . . . . . . . . . 8

3.3 .1 OpenFAST . . . . . . . . . . . . . . . . . . 8

3.3 .2 Fast.Farm Wake Model . . . . . . . . . . . . . . . . . . 10

3.4 Simulation Execution $\ldots \ldots \ldots \ldots \ldots$. . . . . . . . . . . 16

3.4 .1 Uncurtailed Yaw Exploration $\ldots \ldots \ldots . . \ldots . . \ldots 16$

\begin{tabular}{|lll}
\hline & Problem Statement & 19
\end{tabular}

4.1 Proposed Exploration Technique . . . . . . . . . . . . . . . . . . . . 20

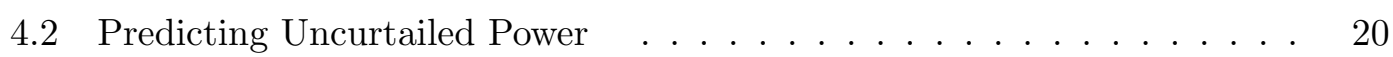


\begin{tabular}{|ll|r}
5 & Results & 23
\end{tabular}

6 Conclusions and Future Work $\quad 27$

6.1 Limitations $\ldots \ldots \ldots \ldots \ldots$. . . . . . . . . . . . . . . . . 27

6.2 Future Work . . . . . . . . . . . . . . . . . . . . . . . . . 28

\begin{tabular}{lr}
\hline References & 30
\end{tabular} 


\section{List of Tables}

3.1 NREL 5-MW Turbine Control Region Parameters . . . . . . . . . . 6

$3.2 \quad$ FAST.Farm Simulation Dimension Inputs . . . . . . . . . . . . . . . 16 


\section{List of Figures}

2.1 Exploration technique for garnering useful yaw wake-steering data while curtailing the power output of a two turbine wind farm. . . . . . . . . . 4

3.1 Wind turbine control regions based on wind speed. . . . . . . . . . . 6

3.2 Upstream turbines have the potential to yaw offset $\left(\gamma_{1}\right)$ to steer their wake deficits away from downstream turbines. This decreases the upstream turbine power production but has shown potential in increasing downstream turbine power output to have a net increase in farm power

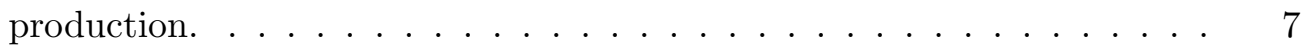

3.3 Blade Element Momentum calculates loads one cross section at a time in order to break down the 3 dimensional physics into 2 dimensional force vectors, lift force, 1 , and drag force, d. The wind "velocity triangle" is shown with incoming wind, $V_{o}$, rotor speed, $\omega$, blade radius, $r$, and axial and tangential induction factors, a and $\mathrm{a}^{\prime}$. . . . . . . . . . 99 9

3.4 The turbine is modelled as a porous actuator disk causing a near wake pressure drop. Mass and momentum balancing requires the radial expansion of the stream-tube as it passes through the disk. free-stream pressure and wind speed, $\mathrm{p}_{\infty}$ and $\mathrm{U}_{\infty}$, waked wind speed, $\mathrm{U}_{W}$, and pressure directly before and after the actuator disc, $\mathrm{p}_{d}^{+}$and $\mathrm{p}_{d}^{-} . \ldots . . .11$

3.5 Wake evolution given a thin shear layer approximation of the NavierStokes equations. [1]. . . . . . . . . . . . . . . 12

$3.6 \quad$ Wake meandering occurs as a consequence of large eddies (diameter $i$ 2 turbine diameters). The quasi-steady-state wake evolution acts as a passive tracer in these large eddies [1]. . . . . . . . . . . . . . . 12 
3.7 Transience of FAST.Farm is achieved via a simple low pass filter applied at the turbine disc wake plane output [1]. . . . . . . . . . . 13

3.8 Incoming wind is deflected to the side by a turbine with yaw offset. This is achieved in the FAST.Farm model by framing each wake as a profile parallel to the rotor disc, retaining the lateral component of the wind velocity in the BEM calculations. . . . . . . . . . . . . . . . . . . . 14

3.9 Turbine location and varying resolution grids for wake modelling in NREL's FAST.Farm simulator software [1]. . . . . . . . . . . . . . 15

3.10 Smoothed pre-generated ambient wind is input into the FAST.Farm simulation. . . . . . . . . . . . . . . . . . . . 17

3.11 Turbine locations and hub height $90 \mathrm{~m}$ wind speed slice for simulated two turbine wind farm simulated in FAST.Farm. This smoothed input wind makes it such that wake meandering is not modelled in these experiments. 18

3.12 Uncurtailed baseline exploration shows power outcomes if a yaw exploration experiment could be done in uncurtailed periods. Total farm power increases by $5.0 \%$ at an upstream turbine yaw angle of 16 degrees. . . . 18

4.1 Turbine controller determines generator torque based on current generator speed. . . . . . . . . . . . . . . . . . . 21

5.1 Power measurements as recorded in FAST.Farm simulation during the exploration experiment as shown in figure 2.1$] \ldots . . . . . . . .23$

5.2 Downstream turbine blade pitch changes to regulate farm power to a constant 1.5MW while the upstream turbine explores to different yaw states . . . . . . . . . . . . . . . . . . . . 24

$5.3 \quad C_{p}$ ratios predict downstream turbine power outputs from curtailed operating states. . . . . . . . . . . . . . . . . 25

$5.4 \quad C_{p}$ ratios predict total farm power from a curtailed farm power, predicting an optimal upstream turbine yaw angle of 14 degrees. . . . . . . . . . 26

$6.1 \quad$ A 3 by 3 wind farm curtailing 3 turbines while wake steering with the remaining upstream turbines. Farms larger than 2 turbines may be able to explore to wake steering states during curtailed periods without needing to pitch the downstream turbine in the pairing, making for a more accurate prediction of uncurtailed power production. . . . . . . . . . . . 29 


\section{Chapter 1}

\section{Overview}

Wind turbine wakes reduce energy output of wind farms. Turbines can steer wakes around their neighbors but sensor and model uncertainty makes this difficult. Physical experiments can be prohibitively costly given turbines will likely produce less power while they find the optimal yaw angle for wake steering.

The novel approach proposed in this thesis utilizes grid curtailment periods, (typically rare) times when wind farms intentionally produce less power than would otherwise be captured. During these times, the upstream turbine(s) can explore yaw angles to find the optimal angle for wake steering while the downstream turbine(s) pitch their blades to keep the farm power at the desired curtailment wattage. The optimal yaw angles found during these curtailment periods can then be applied to all uncurtailed periods which include the vast majority of operation times.

This technique is simple enough for nearly all utility-scale wind farms to execute and addresses one of the major causes of power loss in wind farms. The wide-ranging applicability, usefulness, and novelty of this method speak to the contribution of this thesis. Other research has investigated the use of curtailment periods for load reduction and other research has both investigated and implemented wake steering in wind farms, no investigation of how curtailment periods can improve uncurtailed operation (via wake steering in this research) has been conducted. At present, the author is the sole researcher and discoverer of this approach.

The following chapters give the details of this approach: Chapter 2 briefly presents the history of, and science behind, the subjects presented in this thesis. In Chapter 
3 the experiment is outlined. Chapter 4 describes the simulation process used in the analysis. Chapter 5 follows the chain of reconstruction software used to obtain meaningful results from data. Chapter 6 hashes out the strategy for analysis and presents the data and simulated sets that will be used in the analysis. Chapter 7 demonstrates the implementation of the event selection processes. In Chapter 8 those events selected in Chapter 7 are analyzed. Chapter 9 presents a final discussion of the analyses presented in the thesis. 


\section{Chapter 2}

\section{Intro}

As a wind turbine removes energy from the wind, it produces a wake that disperses downstream. This wake can interact with downstream turbines, often increasing loading moments and decreasing electricity production [2, 3]. Yaw-induced wake-steering seeks to mitigate the negative impacts of wakes on downstream turbines [4]. Unfortunately, physical wind farm experimentation to find the optimal upstream turbine yaw angle can be risky both financially and mechanically [4]. Wake-steering models offer a low-risk method for predicting the optimal upstream turbine yaw angle. However, due to model uncertainty and sensor uncertainty, physical execution of yaw-induced wake steering may be less effective when relying solely on model-based approaches [5, 6]. Grid curtailments, controlled reductions in power production that occur if electrical power production in a grid exceeds electricity demanded, offer times for low-risk physical experimentation with minimal inaccuracies [7]. Curtailment periods may occur while turbines are operating in below-rated wind speeds. Wake steering can only improve total farm power output when in below-rated wind speeds. Therefore, below-rated curtailment experimentation will give the most relevant data for uncurtailed wake steering for power optimization. This low-risk, higher-accuracy method, shown in Figure 2.1, is the topic of this research.

As renewable energy becomes a greater percentage of a grid's overall electricity production, the frequency and magnitude of curtailment periods will likely increase due to the variability of wind and solar resource [8]. Curtailing a wind turbine is typically achieved by pitching a turbine's blades and operating at a non-optimal tip speed ratio. This reduces aerodynamic forces from the wind therefore reducing the amount of energy 

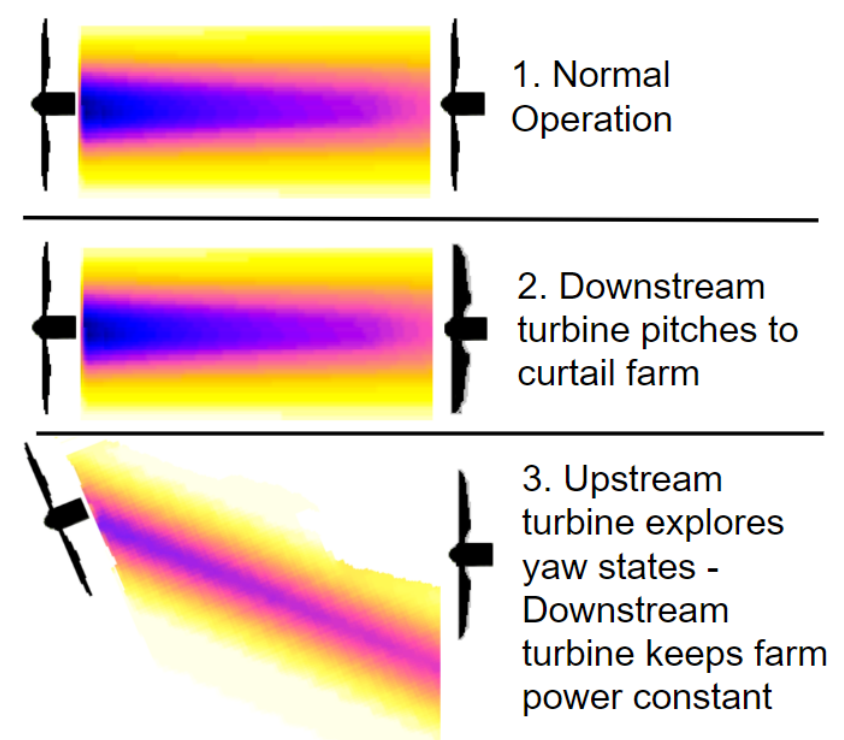

Figure 2.1: Exploration technique for garnering useful yaw wake-steering data while curtailing the power output of a two turbine wind farm.

captured [9].

The topics of wind farm wake effects and grid stabilization via wind farm control is an actively researched topic. Coordinated control of horizontal axis wind turbines in a wind farm has shown benefits compared to independent operation in a number of studies [2, 10, 4]. Yaw-induced wake steering is a promising method that can be implemented on existing utility-scale wind farms [11]. Yaw-induced wake steering works by pointing a wind turbine slightly offset from the wind direction. The vector face of the turbine is the approximate force direction applied to the wind, causing the wake to deflect to the side. This can steer the low-energy wind profile in the wake away from downstream turbines thus leaving higher energy wind for capture. Related work has shown the positive potential of wind farms operation during grid curtailment periods [12] [13] [14] [15. A novel feature of this work relative to the existing literature is how this work seeks to use grid curtailment periods to improve performance during uncurtailed periods. 


\section{Chapter 3}

\section{Background}

\subsection{Individual Turbine Theory}

The majority of this thesis centers around the relation of the turbine operating state and incoming wind to the power captured. The power (W) captured from the wind by a single turbine is given as follows $[9]$ :

$$
P=\frac{1}{2} \rho A U^{3} C_{p}(\beta, \lambda)
$$

where $\rho$ is the air density (set to $\left.1.225 \mathrm{~kg} / \mathrm{m}^{3}\right), A$ is the turbine swept area $\left(\mathrm{m}^{2}\right)$, and $U$ is the incoming wind speed perpendicular to the rotor plane. $(\mathrm{m} / \mathrm{s})$. The efficiency of the wind turbine is denoted by the power coefficient $C_{p}(\beta, \lambda)$ which is controlled by changing the turbine's blade pitch angle $\beta$. It also depends on the (non-dimensional) ratio of the tangential blade tip velocity to the incoming wind speed. This tip speed ratio $\lambda$ is given as follows [9]:

$$
\lambda=\frac{\Omega R}{U}
$$

$\Omega$ is the rotor speed ( $\mathrm{rad} / \mathrm{sec})$ and $R$ is the rotor radius $(\mathrm{m})$.

Figure 3.1 illustrates the typical dependence of power captured versus incoming wind speed $U$. Turbine operation is broken down into four different regions depending on the wind speed. The turbine blade pitch, yaw, and generator torque are operated differently in each region. For context, Table 3.1 shows the parameters for the NREL 5-MW reference turbine [16]. This is the turbine used in the example study below. 
Region 1 describes low wind speeds that are not significant enough to power the wind generator. In this region, the wind turbine may pitch its blades at roughly a 90 degree angle to feather the blades (note the feathering angle for zero lift can vary from turbine to turbine and is not always 90 degrees). This minimizes aerodynamic loads and keeps rotor speed at zero. Region 4 has similar controls objectives to shut down the turbine but for the purpose of load mitigation in high wind speeds.

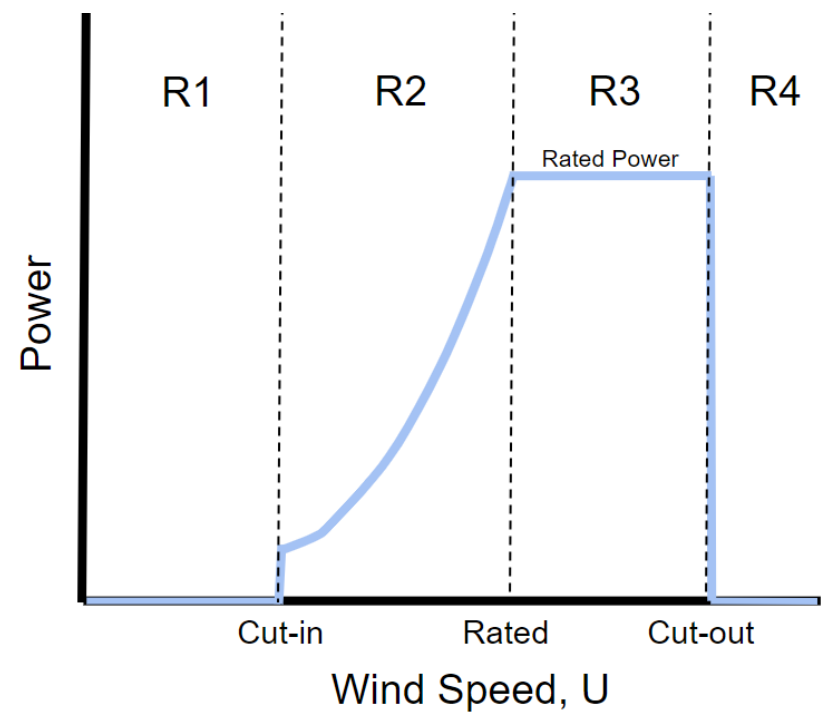

Figure 3.1: Wind turbine control regions based on wind speed.

Table 3.1: NREL 5-MW Turbine Control Region Parameters

\begin{tabular}{|c|c|}
\hline Rated Power & $5 \mathrm{MW}$ \\
Cut-In Wind Speed & $3 \mathrm{~m} / \mathrm{s}$ \\
Rated Wind Speed & $11.4 \mathrm{~m} / \mathrm{s}$ \\
Cut-Out Wind Speed & $25 \mathrm{~m} / \mathrm{s}$ \\
\hline
\end{tabular}

Region 2 describes moderate wind speeds where the control objective is to capture as much power from the wind as possible. It does this by maximizing its power coefficient $C_{p}(\beta, \lambda)$. In this region, the blades are kept at an optimal 0 degree pitch angle $\beta$. The generator controls its torque to keep the turbine at the optimal tip speed ratio $\lambda$. For the NREL 5-MW reference turbine used here the optimal $\lambda$ is 7.55.

Region 3 describes a region of high wind speeds. In this region the turbine limits 
how much power it captures from the wind, attempting to stabilize at its rated power. In this region the wind turbine varies its blade pitch angle to keep the aerodynamic torque on the rotor constant while generator torque attempts to keep the rotor speed constant. It is of note that any increase in wind speed is not ideal in region 3 due to load increases. Therefore, wake steering is not used to increase power capture when the downstream turbine is in region 3 conditions.

\subsection{Wind Farm Theory}

The interactions between wind turbines via wakes require additional modeling. If the wake deficit of the upstream turbine "hits" the downstream turbine it reduces wind speed at the downstream turbine and thus decreases available power. Consider a two turbine farm with the free-stream wind speed of $U$ as shown in Figure 3.2 .

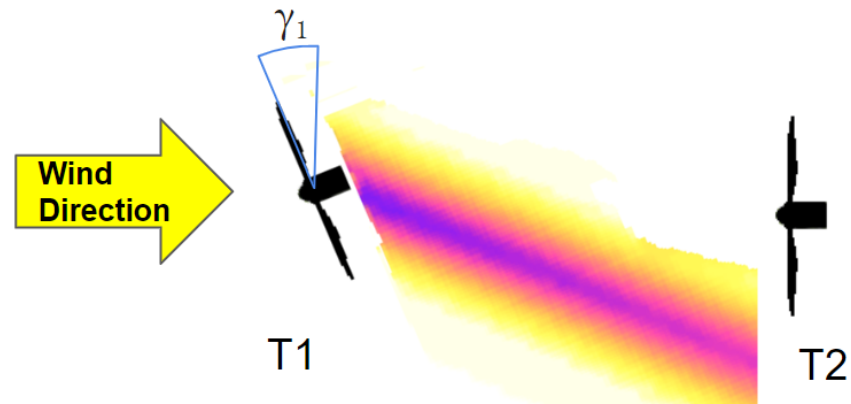

Figure 3.2: Upstream turbines have the potential to yaw offset $\left(\gamma_{1}\right)$ to steer their wake deficits away from downstream turbines. This decreases the upstream turbine power production but has shown potential in increasing downstream turbine power output to have a net increase in farm power production.

Let $\gamma_{1}$ denote the yaw offset angle for the upstream turbine relative to the free-stream wind direction. In addition, label the upstream and downstream turbines as 1 and 2, respectively. Thus $U_{1}\left(\gamma_{1}\right)$ and $U_{2}\left(\gamma_{1}\right)$ are the incoming wind speeds (perpendicular to the rotor plane) at the upstream and downstream turbines. These wind speeds depend on the yaw offset of the upstream turbine. For example, the component of the incoming wind perpendicular to the rotor plane of the upstream turbine is reduced by a factor of $\cos \left(\gamma_{1}\right)$. As a result, the upstream turbine has incoming speed $U_{1}\left(\gamma_{1}\right)=U \cos \left(\gamma_{1}\right)$. It 
follows from Equation 3.1 that the captured power is:

$$
P_{1}\left(\gamma_{1}\right)=\frac{1}{2} \rho A U^{3} \cos ^{3}\left(\gamma_{1}\right) C_{p, \max }
$$

Note the upstream turbine is assumed to maximize its power capture in both the uncurtailed and curtailed yaw explorations. This is the reason for the use of $C_{p, \max }$ in Equation 3.3 .

The yaw offset of the upstream turbine also affects the direction of the downstream wake. Thus the incoming wind speed and power captured by the downstream turbine becomes a function of upstream turbine yaw. Equation 3.1 is modified to explicitly denote this dependence of the downstream power capture on the upstream turbine yaw:

$$
P_{2}\left(\gamma_{1}\right)=\frac{1}{2} \rho A U_{2}\left(\gamma_{1}\right)^{3} C_{p}(\beta, \lambda)
$$

The total power of the two turbine wind farm is simply the sum of the power captured from the upstream and downstream turbines:

$$
P_{\text {farm }}\left(\gamma_{1}\right)=P_{1}\left(\gamma_{1}\right)+P_{2}\left(\gamma_{1}\right)
$$

\subsection{FAST.Farm Model}

To model the turbine operation, and wake interactions in this research, the National Wind Technology Center's FAST.Farm mid-fidelity wind farm simulator was utilized. FAST.Farm utilizes OpenFAST, an open source wind turbine simulation tool that predicts individual turbine loadings and power output using aero-hydro-servo-elastic engineering models [1].

\subsubsection{OpenFAST}

Each individual wind turbine in a FAST.Farm simulation is executed as an independent OpenFAST process. OpenFAST models individual turbine physics as follows:

Aero- OpenFAST's Aerodyn module calculates loads on both the wind turbine's rotor and tower. Blade-Element Momentum (BEM) theory is used for calculating forces on the blades. BEM analyzes loads on individual cross section elements of the blades as shown in figure 3.3 . 


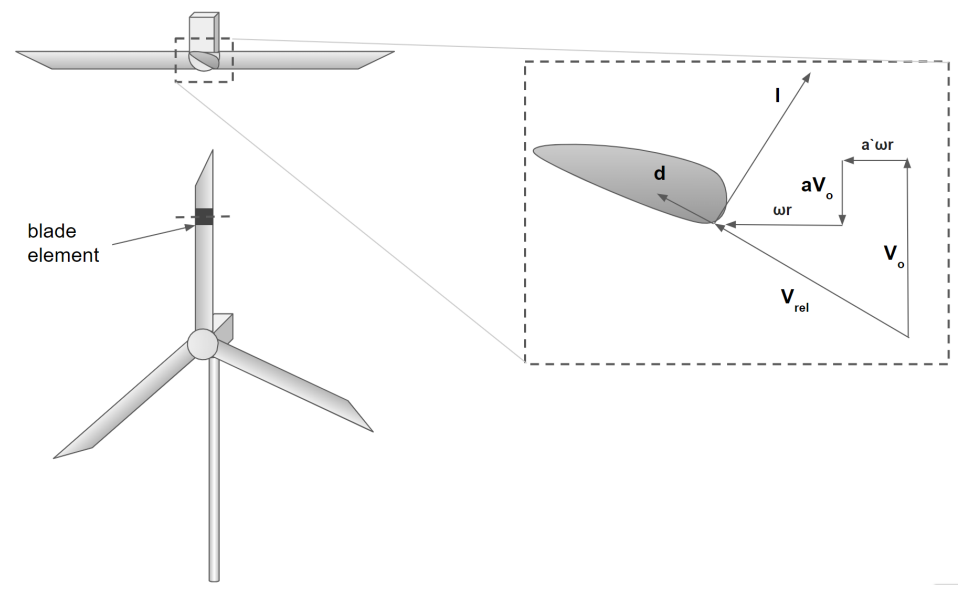

Figure 3.3: Blade Element Momentum calculates loads one cross section at a time in order to break down the 3 dimensional physics into 2 dimensional force vectors, lift force, l, and drag force, d. The wind "velocity triangle" is shown with incoming wind, $V_{o}$, rotor speed, $\omega$, blade radius, $r$, and axial and tangential induction factors, a and $\mathrm{a}^{\prime}$.

Analyzing one slice at a time allows for changing 3 dimensional physics into simpler 2 dimensional vectors as shown. Lift and drag forces are calculated using a lift coefficient table and drag coefficient table for the given airfoil cross section as calculated in equations 3.6 and 3.7 . 


$$
\begin{gathered}
l=\frac{1}{2} \rho V_{r e l}^{2} c C_{l}(\alpha, R e) \\
d=\frac{1}{2} \rho V_{r e l}^{2} c C_{d}(\alpha, R e)
\end{gathered}
$$

The forces are calculated from equations 3.6 and 3.7 with air density, $\rho$, chord length, c, coefficients of lift and drag, $C_{l}$ and $C_{d}$, angle of attack, $\alpha$ and Reynolds number, Re. The forces from each cross section are applied to the blade as a whole via the actuatorline model. This means the forces are applied to a line that acts as the model for the blade. Rotor torque is calculated from these forces along the actuator-line, giving the power generated by the turbine utilized in this research.

Servo- OpenFAST's Servodyn module allows for controlling the turbine's blade pitch motor, yaw motor, and generator torque. Further details of the controllers used in this research can be found in the Approach chapter.

Elastic- OpenFAST's Beamdyn module calculates stresses and bending mechanics on the blades of the wind turbine. It utilizes the forces calculated by the Aerodyn module.

Other- OpenFAST also includes modules for blade icing and a hydrodynamic model for offshore wind farm applications as well as other modules that were not relevent to this research but should be noted.

\subsubsection{Fast.Farm Wake Model}

FAST.Farm adds a farm-wide atmospheric boundary layer physics module to model wake deficits, advection, deflection, meandering, and merging as a result of wind turbine interaction. Some of the aspects of the Dynamic Wake Meandering (DWM) model make up the wake model of FAST.Farm primarily for wake-deficit evolution and wake meandering [17]. Each turbine has its own Wake Dynamics (WD) module for calculating its quasi-steady state wake deficit, deflection, and meandering. One single Ambient Wind and Array Effects (AWAE) module processes ambient wind and merges overlapping wakes across the wind farm. 
The DWM quasi-steady state wake deficit behind a turbine is based on simplifying the wind turbine into a porous actuator disc that slows down the wind speed and causes a pressure drop as show in figure 3.4. Assuming an incompressible flow, a basic mass and momentum balance leads to an expanding stream-tube. Farther downstream as turbulent mixing brings outside momentum into the stream-tube, the Gaussian wake deficit recovers to the free stream wind speed.

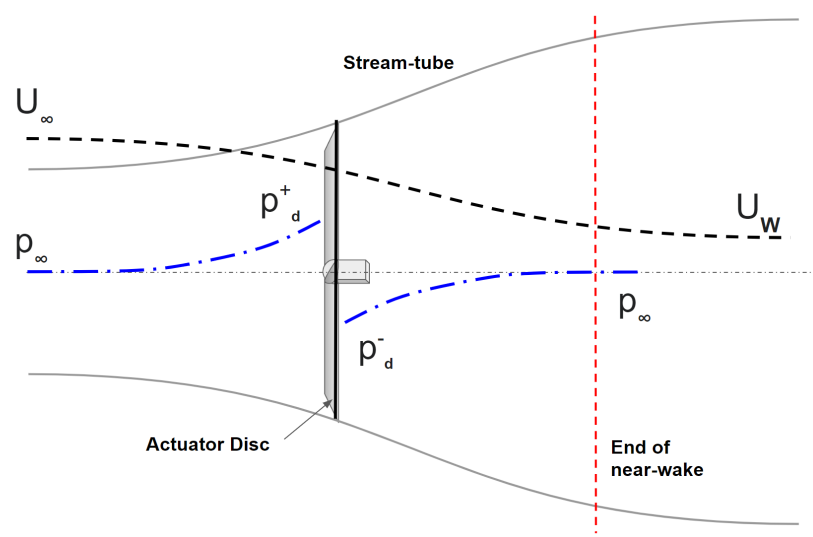

Figure 3.4: The turbine is modelled as a porous actuator disk causing a near wake pressure drop. Mass and momentum balancing requires the radial expansion of the stream-tube as it passes through the disk. free-stream pressure and wind speed, $\mathrm{p}_{\infty}$ and $\mathrm{U}_{\infty}$, waked wind speed, $\mathrm{U}_{W}$, and pressure directly before and after the actuator disc, $\mathrm{p}_{d}^{+}$and $\mathrm{p}_{d}^{-}$.

This wake-deficit evolution is modelled using the thin shear-layer approximation of the Navier-Stokes equations. This forms an axisymetric wake-deficit evolution of the form shown in figure 3.5. The rate of recovery for this wake is determined by turbulence and ambient small eddies with diameters less than 2 turbine diameters. Rate of recovery is also modelled by taking local spatial averages between ambient wind and wake deficit. A near-wake correction is applied at the rotor disk to account for vortex shedding and other factors that make the first 2-3 diameters downstream not conform to the disc model used in the thin shear-layer Navier-Stokes equations. This quasi-steady state wake deficit is then meandered by treating the wake as a passive tracer of turbulent eddies with diameters greater than 2 turbine diameters as shown in figure 3.6. These turbulent eddies are processed into WD via AWAE from a pre-generated Large Eddy 
Simulation (LES) which can be more accurate than synthetic turbulence. After WD increments the wake downstream and applies the meandering, the AWAE module then finds where wakes overlap and applies a simple root-sum-squared method.

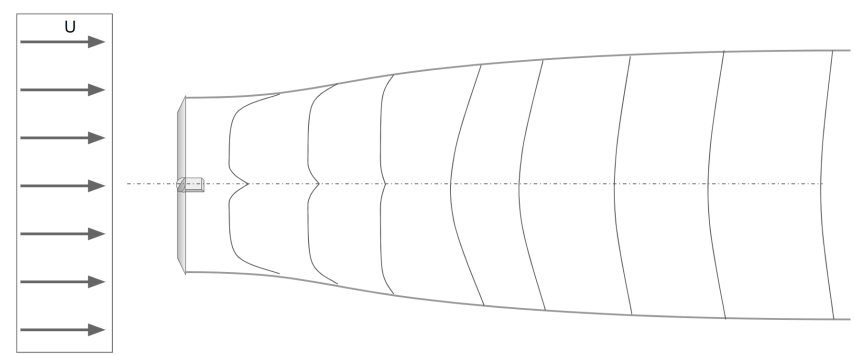

Figure 3.5: Wake evolution given a thin shear layer approximation of the Navier-Stokes equations. [1].
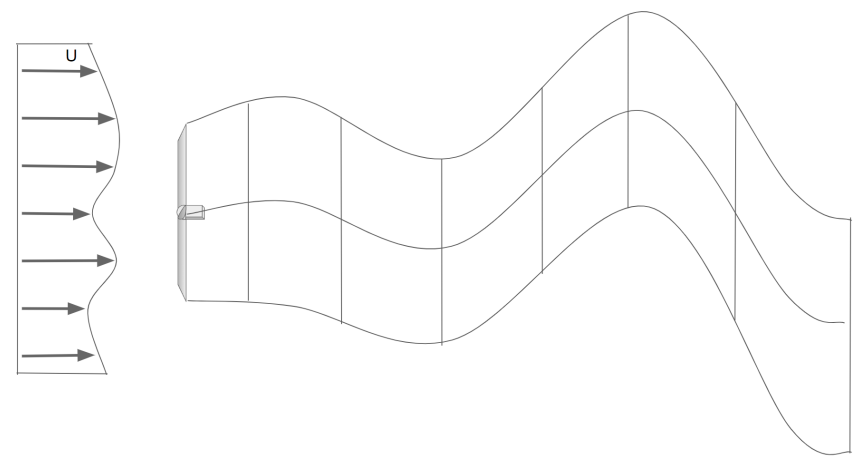

Figure 3.6: Wake meandering occurs as a consequence of large eddies (diameter i, 2 turbine diameters). The quasi-steady-state wake evolution acts as a passive tracer in these large eddies [1].

In order to model the inertia of changing wind turbine operation and wind interaction, a single-pole low pass filter acts at the turbine disc as a wake plane is being produced. This allows for these quasi-steady state wake profiles to join together in a way that resembles the transient wake that occurs during changing turbine states or incoming wind conditions as seen in figure 3.7 . 


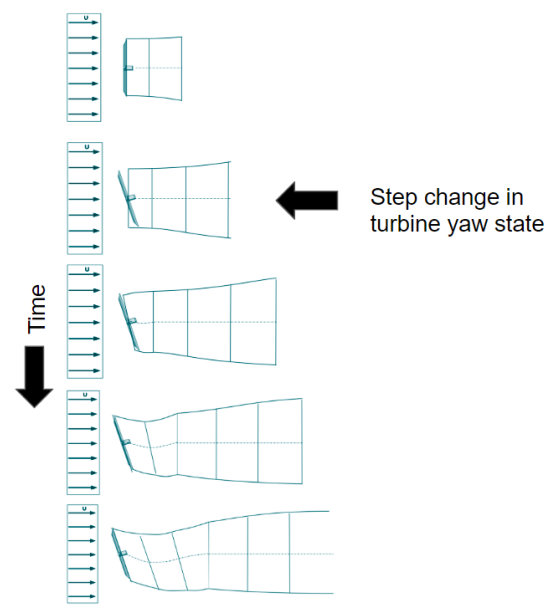

Figure 3.7: Transience of FAST.Farm is achieved via a simple low pass filter applied at the turbine disc wake plane output [1]. 
A key enhancement included in the FAST.Farm WD module that was added to the DWM model is wake deflection as seen in figure 3.8. By propagating wakes as profiles parallel to the rotor disk- as opposed to being perpendicular profiles to wind directionthe BEM simplification allows for the retention of the lateral component of the wind vector.

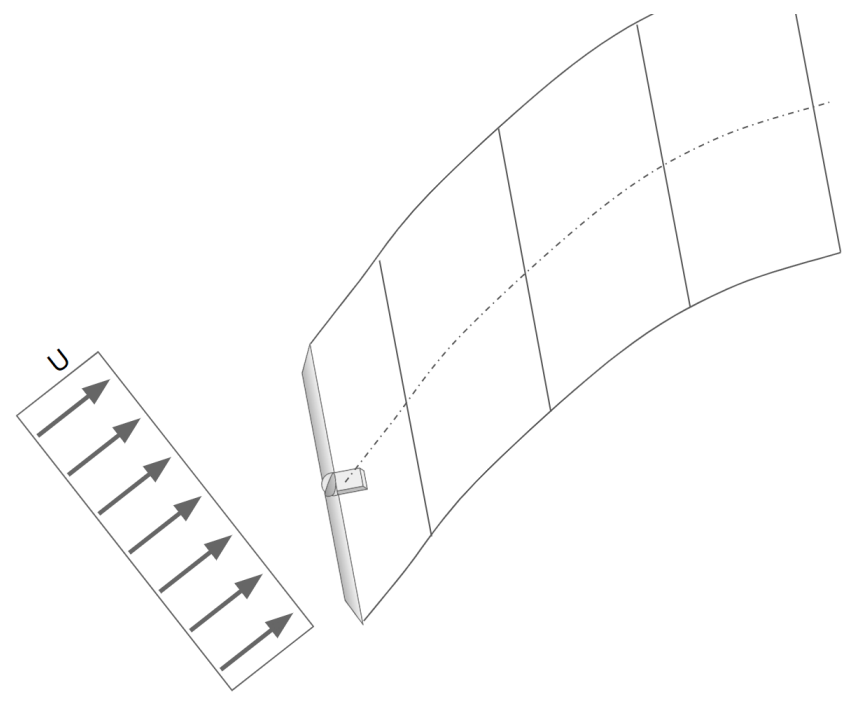

Figure 3.8: Incoming wind is deflected to the side by a turbine with yaw offset. This is achieved in the FAST.Farm model by framing each wake as a profile parallel to the rotor disc, retaining the lateral component of the wind velocity in the BEM calculations. 


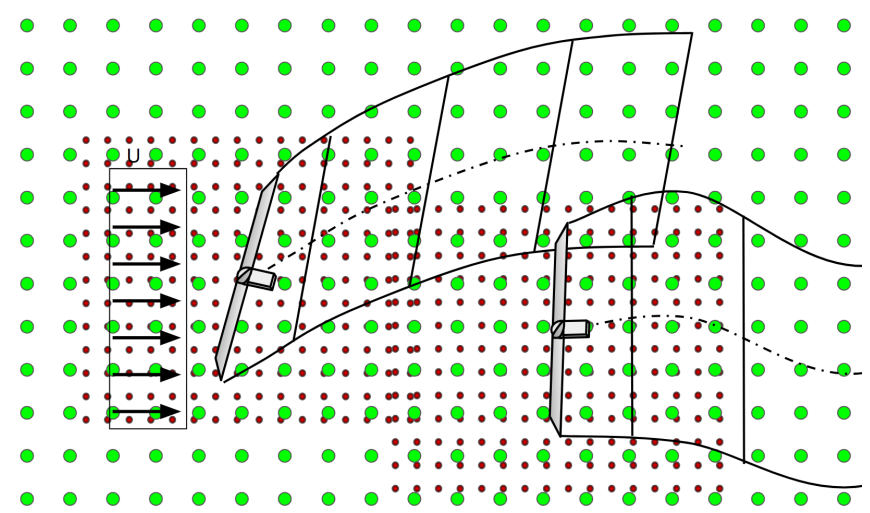

Figure 3.9: Turbine location and varying resolution grids for wake modelling in NREL's FAST.Farm simulator software [1].

FAST.Farm models winds at varying resolutions: a high resolution for the volumes closest to wind turbines and a lower resolution encompassing the entire wind farm. FAST.Farm takes in the pre-generated LES wind velocity vectors represented by each dot as shown in Figure 3.9. These wind velocity vectors describe how the winds in the array would be changing in time if no wind turbines were present in the flow field. The higher fidelity arrays around each wind turbine allow for the more complex near-wake corrections and BEM analysis.

FAST.Farm has been calibrated and validated against SOWFA, a high-fidelity largeeddy simulation tool that also finds wind turbine loading and power outputs [18, 19]. 


\subsection{Simulation Execution}

This research focuses on a theoretical two turbine wind farm comprised of two NREL 5-MW wind turbines. An $8 \mathrm{~m} / \mathrm{s}$ wind was simulated traveling from west to east as shown in Figure 3.11. The farm was modeled with low and high resolution wind vector input volumes with physical dimensions (length, width, and height), spacing between grid points, and calculation time steps as given in Table 3.2

\begin{tabular}{|c|c|c|}
\hline$x$ dimension & Low Resolution & High Resolution \\
$y$ dimension & $5000 \mathrm{~m}$ & $160 \mathrm{~m}$ \\
$z$ dimension & $2000 \mathrm{~m}$ & $160 \mathrm{~m}$ \\
spacing & $350 \mathrm{~m}$ & $170 \mathrm{~m}$ \\
time step & $10 \mathrm{~m}$ & $10 \mathrm{~m}$ \\
\hline
\end{tabular}

Table 3.2: FAST.Farm Simulation Dimension Inputs

Here, both the low and high resolution grids follow a basic power law equation with each grid point having the same velocity as the grid points at the same height as shown by the input slice in figure 3.10. This smoothed input wind makes it such that wake meandering is not modelled in these experiments. The vertical shear power law for wind speed is given as [9]:

$$
\frac{u}{u_{r}}=\left(\frac{z}{z_{r}}\right)^{\alpha}
$$

where $u$ is the wind speed at a given height $z$. The reference wind speed $u_{r}$ is given as $8.0 \mathrm{~m} / \mathrm{s}$ at a hub height $z_{r}$ of $90 \mathrm{~m}$. This yields the hub height wind speeds shown in Figure 3.11. For this wind profile a standard $\alpha$ of 0.14 represents the increase of wind speed as height increases.

\subsubsection{Uncurtailed Yaw Exploration}

This section describes an uncurtailed yaw exploration method. This provides a baseline for comparison to the proposed method to explore during curtailed operation. For this baseline, the upstream turbine yaw angle $\gamma_{1}$ is held constant for 2000 seconds during an uncurtailed operation. The yaw angle is then increased by 2 degrees and the simulation is repeated with the same incoming wind profile and all other quantities kept constant. 


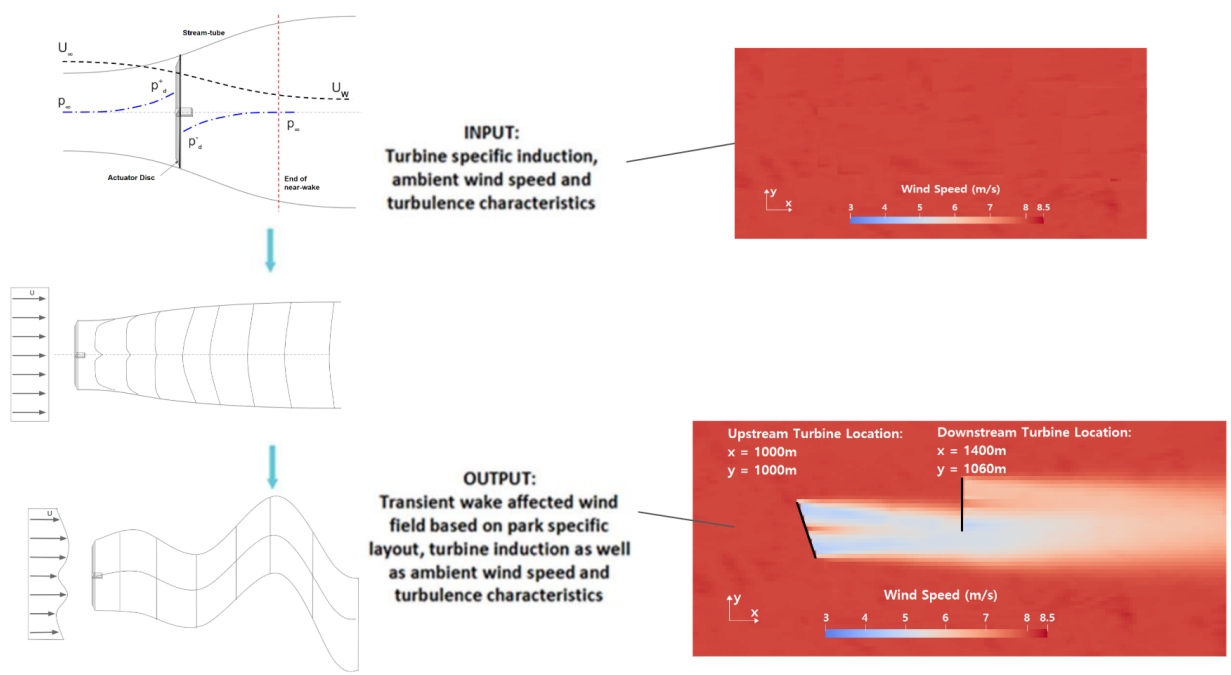

Figure 3.10: Smoothed pre-generated ambient wind is input into the FAST.Farm simulation.

This is done for $\gamma_{1}$ from 0 to 28 degrees. Figure 3.12 shows the power captured by the upstream turbine, downstream turbine, and farm power. The upstream power output, $P_{1}\left(\gamma_{1}\right)$, varies with the yaw angle as $\cos ^{3}\left(\gamma_{1}\right)$. This is consistent with the expected relationship given in Equation 3.3. At small (positive) yaw angles, the power loss by the upstream turbine is less than the power decrease at the downstream turbine. This is due to the upstream turbine deflecting its wake to the side to avoid the downstream turbine. As $\gamma_{1}$ increases past 16 degrees, the increase in downstream turbine power is no longer greater than the loss of power for the upstream wind turbine. The optimal yaw angle that maximizes power production is $\gamma_{1}^{*}=16^{\circ}$. This increases power production by $5.0 \%$ compared to a farm with no yaw-induced wake steering in these wind conditions. 


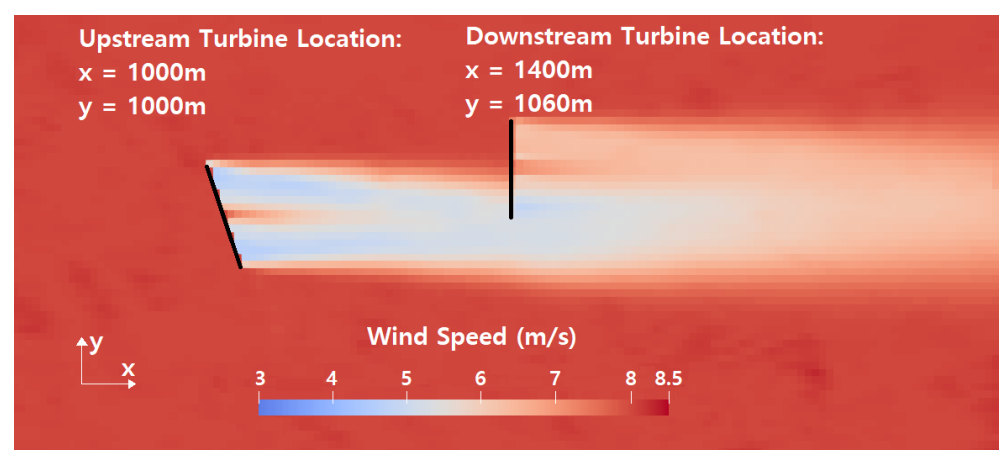

Figure 3.11: Turbine locations and hub height $90 \mathrm{~m}$ wind speed slice for simulated two turbine wind farm simulated in FAST.Farm. This smoothed input wind makes it such that wake meandering is not modelled in these experiments.

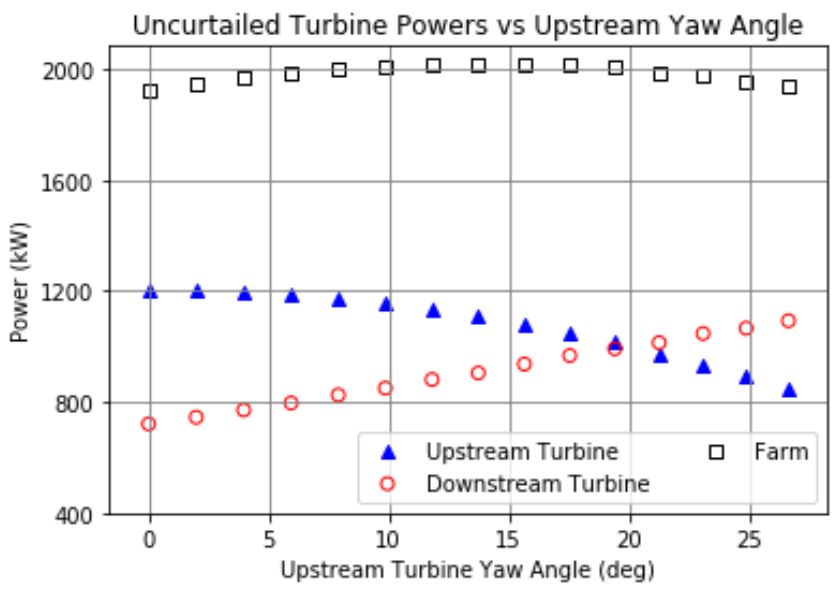

Figure 3.12: Uncurtailed baseline exploration shows power outcomes if a yaw exploration experiment could be done in uncurtailed periods. Total farm power increases by $5.0 \%$ at an upstream turbine yaw angle of 16 degrees. 


\section{Chapter 4}

\section{Problem Statement}

The previous section summarized an approach to determine the optimal upstream turbine yaw angle from exploration during uncurtailed periods. This approach has both financial and mechanical risks. If the upstream turbine had explored to yaw angles that were ineffective at wake steering, the farm would have produced less electricity for an extended period of time, equating to lost revenue. Many wind farm owners, like many utility owners, operate with thin profit margins, making failed exploration time too high of cost to justify. Farm-site wind profiles can change over time with changing landscapes or prevailing wind direction and speeds, meaning even if explorations are successful they may need to be executed multiple times through a wind farm's life cycle. Another risk of the uncurtailed method is that it holds the downstream turbine blade pitch at 0 degrees, increasing the aerodynamic loads that occur as a result of waked wind profiles. These wake profiles may be weighted to one side of the wind turbine during upstream turbine yaw exploration.

The financial and mechanical risks can be mitigated by exploring upstream turbine yaw values during curtailment periods. Curtailment periods require the downstream turbine to pitch its blades to nonzero angles for the purpose of controlling farm power production. Pitched blades capture less wind and therefore be subjected to lower structural loads from upstream wakes [20]. Finally, the goal of wind turbine controllers during curtailment periods is to reduce the efficiency of a wind farm. Hence there would be little to no loss of power production if yaw exploration failed to find a beneficial yaw angle. A key technical challenge with this approach is that it requires an estimate of 
uncurtailed power output using data obtained during curtailed periods. The following section demonstrates that this prediction can be done with minimal information: a reliable $C_{p}$ table for the wind turbine in use and accurate measurements of blade pitch, rotor speed, turbine yaw, and turbine power.

\subsection{Proposed Exploration Technique}

The proposed exploration during curtailment is performed as follows. The turbines initially operate normally by maximizing their own power capture. At some point, a signal is provided by the system operator to curtail the farm power output (in this research: from 1.9 MW down to 1.5 MW). The upstream turbine moves from $\gamma_{1}=0^{\circ}$ in the direction predicted to increase power by a computer model, i.e. in the direction of the optimal offset. Throughout the exploration, the downstream turbine continues to hold the total farm power constant using blade pitch control. The power measurements from the upstream turbine are assumed to be available to the downstream turbine controller. The desired power for the downstream turbine $P_{2, \text { desired }}$ is the difference between the desired (curtailed) power for the farm minus the power produced by the upstream turbine. The desired downstream generator torque $\tau_{2}$ is determined from its rotor speed $\omega_{2}$ as shown in in Figure 4.1. The desired rotor speed can then be determined by solving the power equation $P_{2, \text { desired }}=\tau_{2}\left(\omega_{2}\right) \omega_{2}$ for $\omega_{2}$ where $\tau_{2}\left(\omega_{2}\right)$ represents generator torque as a function of generator speed. Finally, the downstream turbine tracks this desired rotor speed by varying blade pitch. In this thesis only collective blade pitch was used but an individual pitch controller could likely be utilized as well.

\subsection{Predicting Uncurtailed Power}

The exploration is performed during a curtailed period. The power produced by the farm, upstream, and downstream turbines during curtailment exploration are thus related by: $\quad \mathrm{P}_{\text {farm }, c}\left(\gamma_{1}\right)=P_{1, c}\left(\gamma_{1}\right)+P_{2, c}\left(\gamma_{1}\right)$

The subscript $c$ describes curtailed operation and, in contrast, $u$ will be used below to denote uncurtailed operation. The downstream turbine uses blade pitch to maintain 


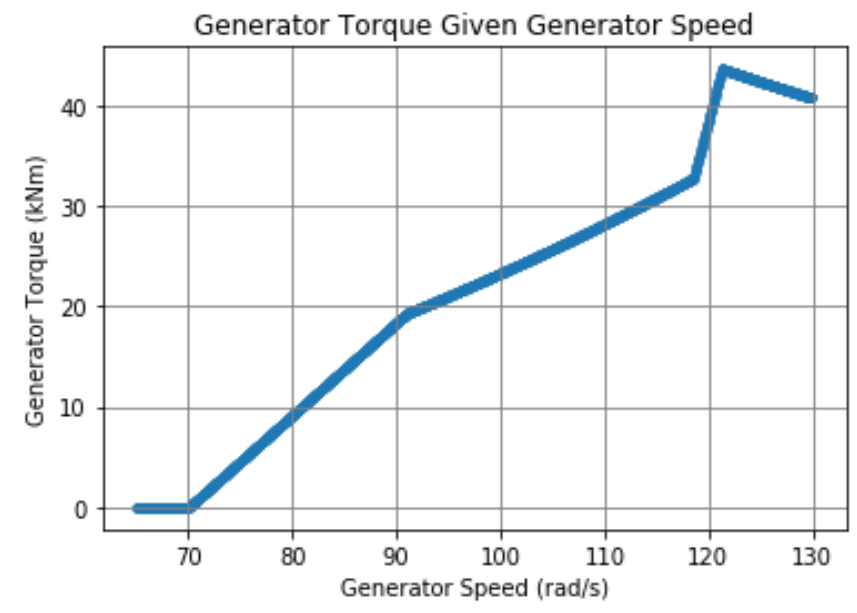

Figure 4.1: Turbine controller determines generator torque based on current generator speed.

the curtailed total farm power demand while the upstream turbine performs yaw exploration. Thus $P_{2, c}\left(\gamma_{1}\right)$ compensates for changes in $P_{1, c}\left(\gamma_{1}\right)$ to ensure that $P_{\text {farm }, c}\left(\gamma_{1}\right)$ remains near the (constant) desired value.

The objective is to use this exploration during curtailment to determine the optimal upstream yaw offset to maximize total uncurtailed power. First note that the upstream turbine is assumed to operate at its peak power coefficient during both curtailed and uncurtailed periods. Hence $P_{1, c}\left(\gamma_{1}\right)=P_{1, u}\left(\gamma_{1}\right)$. However, the downstream turbine is operated differently during the curtailed and uncurtailed periods. The power produced during curtailment must be used to estimate the uncurtailed power. This is done as follows. First, note that the downstream curtailed power is given as:

$$
P_{2, c}\left(\gamma_{1}\right)=\frac{1}{2} \rho A U_{2}\left(\gamma_{1}\right)^{3} C_{p}\left(\beta_{c}, \frac{R \Omega_{c}}{U_{2}\left(\gamma_{1}\right)}\right)
$$

The downstream velocity $U_{2}\left(\gamma_{1}\right)$ depends on the yaw offset of the upstream turbine but it is the same for both curtailed and uncurtailed operation. This follows because the upstream turbine operates at $C_{p, \max }$ in both cases.

The power $P_{2, c}\left(\gamma_{1}\right)$, blade pitch $\beta_{c}$ and rotor speed $\Omega_{c}$ can be measured during curtailed exploration. This leaves the effective wind speed $U\left(\gamma_{1}\right)$ as the remaining 
unknown 11 This effective wind speed $U\left(\gamma_{1}\right)$ can be estimated from Equation 4.1 using a lookup table for the power coefficient $C_{P}$. For this work, a look-up table for the NREL 5MW turbine was constructed using Aerodyn 15 [21]. The $C_{p}$ lookup-table takes $\beta$ and $\lambda$ as inputs and outputs the $C_{p}$ value. Note, the use of a model-created $C_{p}$ table signifies this approach is not entirely free of wind turbine modeling. However, the aerodynamic modelling of wind turbine blades is likely more accurate and consistent than wake prediction models, which are made up of aerodynamic models and fluid mechanics models. A prediction $\hat{P}_{2, u}\left(\gamma_{1}\right)$ for the uncurtailed power produced by the downstream is obtained assuming maximum power production:

$$
\hat{P}_{2, u}\left(\gamma_{1}\right)=\frac{1}{2} \rho A U_{2}\left(\gamma_{1}\right)^{3} C_{p, \max }
$$

A model-based estimate for $C_{p, \max }$ may be used in the equation above. Alternatively, it can be estimated from data collected during uncurtailed operation. Specifically, the power produced by the upstream turbine during uncurtailed operation at zero yaw offset is:

$$
P_{1, u}\left(\gamma_{1}=0\right)=\frac{1}{2} \rho A U_{1}^{3} C_{p}\left(\beta=0, \frac{\Omega_{u} R}{U_{1}}\right)
$$

As before, this can be used to estimate the effective wind speed $U$ from measurements of power, blade pitch, and rotor speed. The maximum power coefficient is found simultaneously in this process.

Finally, a prediction for the total power produced by the farm during uncurtailed operation is: $\quad \hat{P}_{\text {farm }, u}\left(\gamma_{1}\right)=P_{1, u}\left(\gamma_{1}\right)+\hat{P}_{2, u}\left(\gamma_{1}\right)$ This estimate is formed by exploring with different upstream yaw offsets during curtailment. The optimal yaw offset for uncurtailed operation is obtained by maximizing this predicted power.

\footnotetext{
1 An anemometer is typically installed on the nacelle. However, this is a single hub height measurement in disrupted flow behind the rotor plane.
} 


\section{Chapter 5}

\section{Results}

The curtailment exploration experiment was executed as described in Section 4 . Figure 5.1 shows the power produced from the upstream turbine, downstream turbine, and their summed farm power at each upstream turbine yaw angle. As expected, the upstream turbine generates less power as it yaws farther from the incoming wind direction. The downstream turbine is controlled in a way that keeps total farm power at $1500 \mathrm{~kW}$.

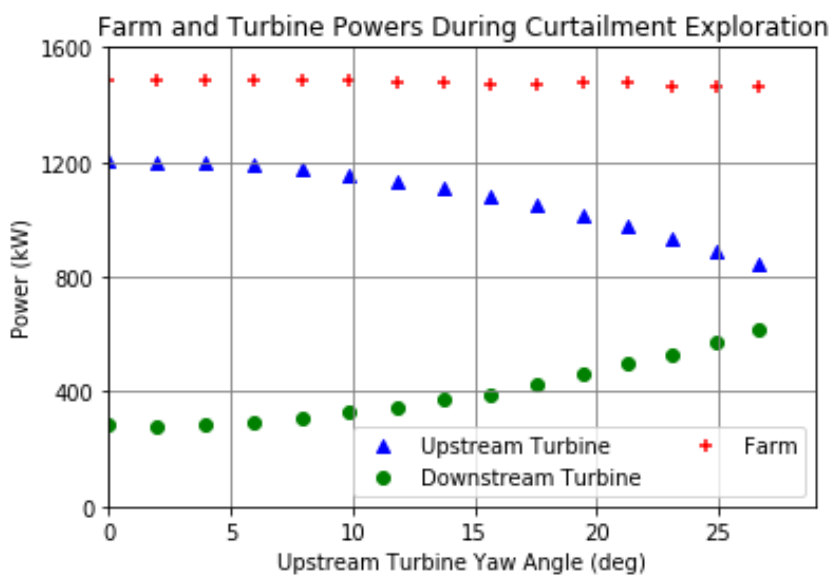

Figure 5.1: Power measurements as recorded in FAST.Farm simulation during the exploration experiment as shown in figure 2.1.

The downstream turbine regulates total farm power by varying its blade pitch angle as shown in Figure 5.2. Note that the upstream turbine yaw angle corresponding to the maximum downstream blade pitch is not the yaw angle that will optimize power 
under normal uncurtailed farm operation. The maximum blade pitch signifies the largest change in how much energy is captured from the wind comparing curtailed to uncurtailed states. However, as the upstream turbine yaw angle increases, the power in the wind also increases. Here, the upstream yaw angle of 10 degrees forces the maximum downstream blade pitch, but the maximum farm power output is predicted to occur at 14 degrees. This small difference between predicted and actual optimal yaw angle is likely due to the $C_{p}$ table not being as accurate at predicting the efficiency of a partially waked wind turbine. Small inconsistencies between simulations may also be present. A $C_{p}$ table for varying incoming wind profiles that exhibit high horizontal shear due to wakes may help to increase prediction accuracy. A smaller step between yaw angles or a nonlinear optimization gradient descent may allow for greater precision by simply taking more data points near the optimal yaw angle.

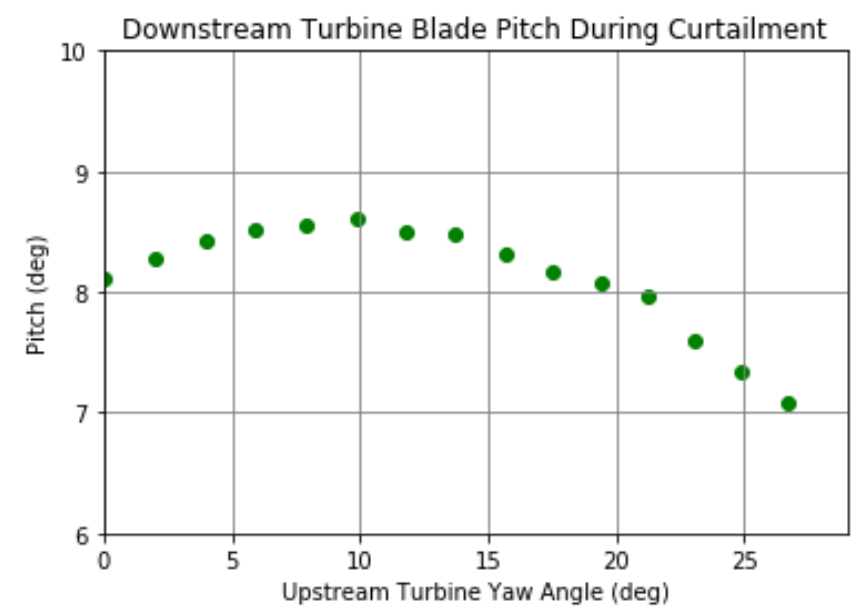

Figure 5.2: Downstream turbine blade pitch changes to regulate farm power to a constant $1.5 \mathrm{MW}$ while the upstream turbine explores to different yaw states.

Although the maximum blade pitch angle does not occur at the optimal upstream yaw angle, in many cases such as the one described in this research, it can signify that the optimal yaw angle must be greater than the yaw angle that caused the maximum downstream blade pitch. If Figure 5.2 was the only exploration data given, a wind farm operator could reasonably assume the optimal upstream turbine yaw angle is greater than 10 degrees.

Figure 5.3 shows the power produced by the downstream turbine during exploration 


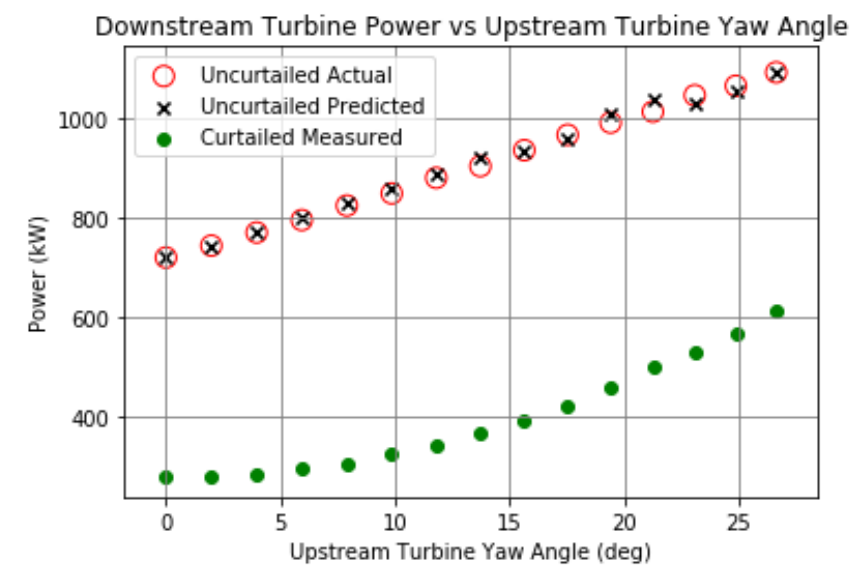

Figure 5.3: $C_{p}$ ratios predict downstream turbine power outputs from curtailed operating states.

(green dots) at various upstream yaw offsets. This figure also shows the power produced during uncurtailed operation along with the prediction as described in the previous subsection. The far left orange circle in Figure 5.3 is assumed to be a known quantity since it represents an uncurtailed farm comprised of a 0 degree upstream turbine yaw angle and a 0 degree downstream turbine pitch. Equation 4.3 gives an uncurtailed $C_{p}$ $=0.45$, a small but significant adjustment to the theoretical maximum efficiency for the NREL $5 \mathrm{MW}$ turbine given as 0.48. Equation 4.2 is then solved for all other yaw angles to predict downstream turbine power if it was not curtailed using blade pitch control.

The predicted downstream power is then added to the upstream turbine measured power output to produce a predicted farm power as a function of upstream yaw angle as shown in Figure 5.4. As noted, the optimal yaw angle for power production does not correspond with the downstream turbine maximum blade pitch angle. Overall, this method shows promise in predicting farm power as a function of upstream turbine yaw angle with relatively few sensors. 


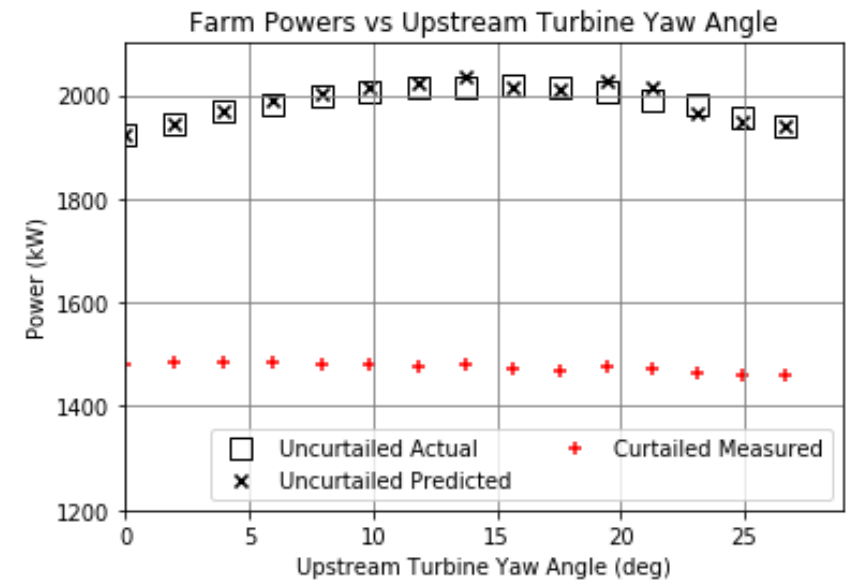

Figure 5.4: $C_{p}$ ratios predict total farm power from a curtailed farm power, predicting an optimal upstream turbine yaw angle of 14 degrees. 


\section{Chapter 6}

\section{Conclusions and Future Work}

Grid curtailment periods offer an opportunity to explore possible wake-steering states to find the optimal yaw angle for maximum power production with minimal mechanical and financial risks. A simple rotor effective wind speed can calculate $C_{p}$ ratios utilized in predicting uncurtailed power from curtailed power. An optimal upstream turbine yaw angle of 14 degrees was predicted from the curtailment exploration with the true optimal angle was 16 degrees. Accuracy may increase if different $C_{p}$ tables are utilized for each magnitude of horizontal wind shear due to partial waking of the downstream turbine. This method requires only blade pitch angle, rotor speed, and yaw angle measurements on the wind turbines. However, This method requires a 5-10 minute duration of constant wind speed and direction.

\subsection{Limitations}

A number of simplifications and assumptions were made in this thesis that may benefit from further experiments and theory. This method requires an accurate $\mathrm{Cp}$ table. How accurate can empirically derived $\mathrm{Cp}$ tables be? What precision is required? Empirically derived $\mathrm{Cp}$ tables are imperfect due to being reliant on nacelle wind sensors that are impacted by blade shadows etc but if these tables are being applied in similar conditions this may prove to be less of a problem. The incoming wind profile was significantly smoothed for this thesis, erasing any wake meandering. Are the predictions as accurate when there's less smoothing and more meandering? 
Can inconsistent wind speeds or directions still garner information about potential wake steering angles or effectiveness? Quantifying power loss in these unstable conditions may also be important. Even when wake steering is possible on a site there may be a failure percentage where a turbine attempts to wake steer but the winds change too drastically to increase power production. Quantifying failure percentage due to unpredictable winds and the resulting power loss would help determine if the average wake steering session at a given wind site would be successful.

\subsection{Future Work}

Future work will look to use similar wake-steering exploration techniques during other times when wind turbines are controlled to work less efficiently such as in wind regions 1 and 3. Larger wind farms will be simulated and analyzed to show how these exploration techniques may be executed with deep-array effects as shown in figure6.1. Wind farms larger than 2 turbines allow for wake steering exploration during curtailment that doesn't require all downstream turbines to pitch their blades. This means some turbine pairings may be operating exactly the same (without pitching their blades to feather) in these curtailed periods as uncurtailed periods. Since these turbines are operating exactly how they would in uncurtailed periods, there would be no dependence on Cp tables. The only requirement would be a consistent wind speed and direction. It is not clear whether this method is limited to collective blade pitch or may also be effective for individual pitch controlled blades. An experiments may be run to discover the feasibility.

Loadings will be analyzed to assess how different yaw angles impact fatigue stresses on both the yawing turbine and the downstream turbine. The change in loads for the downstream turbine as it pitches its blades during curtailment exploration is also of interest for future work. 


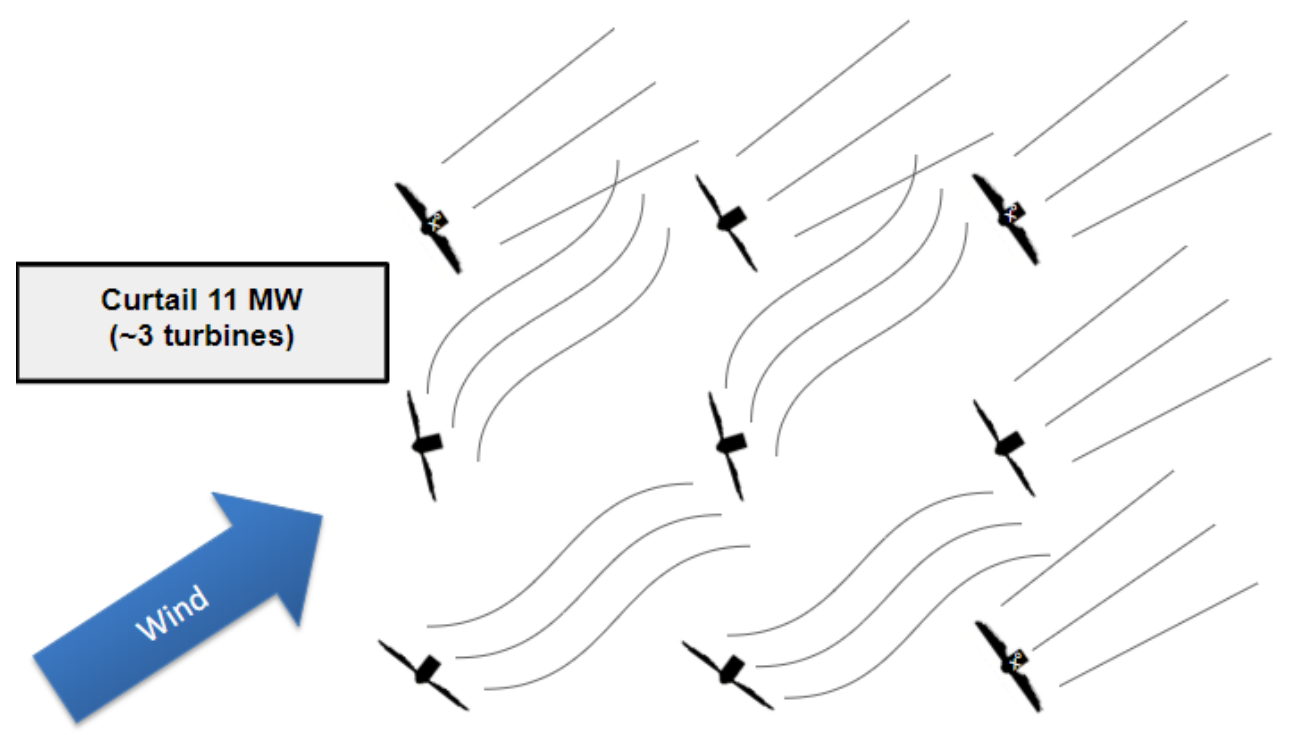

Figure 6.1: A 3 by 3 wind farm curtailing 3 turbines while wake steering with the remaining upstream turbines. Farms larger than 2 turbines may be able to explore to wake steering states during curtailed periods without needing to pitch the downstream turbine in the pairing, making for a more accurate prediction of uncurtailed power production. 


\section{References}

[1] Jason M. Jonkman, Jennifer Annoni, Greg Hayman, Bonnie Jonkman, and Avi Purkayastha. Development of fast.farm: A new multi-physics engineering tool for wind-farm design and analysis. In 35th Wind Energy Symposium, 2017.

[2] S. Boersma, B. Doekemeijer, P. Gebraad, P. Fleming, J. Annoni, A. Scholbrock, J. Frederik, and J.W. van Wingerden. Tutorial on control-oriented modeling and control of wind farms. In American Control Conference, pages 1-18, 2017.

[3] Kenneth Thomsen and Poul Sorensen. Fatigue loads for wind turbines operating in wakes. Journal of Wind Engineering and Industrial Aerodynamics, 80(1):121136, 1999.

[4] Mike T. van Dijk, Jan-Willem van Wingerden, Turaj Ashuri, and Yaoyu Li. Wind farm multi-objective wake redirection for optimizing power production and loads. Energy, 121:561-569, 2017.

[5] Julian Quick, Jennifer Annoni, Ryan King, Katherine Dykes, Paul Fleming, and Andrew Ning. Optimization under uncertainty for wake steering strategies. Journal of Physics: Conference Series, 854, 2017.

[6] John D. Sorensen and Henrik S. Toft. Probabilistic design of wind turbines. Energies, $3(2): 241-257,2010$.

[7] J. Hoyt and P. Seiler. Wind farm wake-steering exploration during grid curtailment. In American Controls Conference, pages 4065-4070, 2020. 
[8] Jacob P. Aho, Andrew D. Buckspan, Fiona M. Dunne, and Lucy Y. Pao. Controlling wind energy for utility grid reliability. Mechanical Engineering Magazine Select Articles, 135(09):S4-S12, 2013.

[9] Nicholas Jenkins, A Burton, D Sharpe, and E Bossanyi. Wind Energy Handbook. John Wiley \& Sons Ltd, United Kingdom, 2001.

[10] Michael F. Howland, Sanjiva K. Lele, and John O. Dabiri. Wind farm power optimization through wake steering. Proceedings of the National Academy of Sciences, 116(29):14495-14500, 2019.

[11] Paul Fleming, Pieter M.O. Gebraad, Sang Lee, Jan-Willem van Wingerden, Kathryn Johnson, Matt Churchfield, John Michalakes, Philippe Spalart, and Patrick Moriarty. Simulation comparison of wake mitigation control strategies for a two-turbine case. Wind Energy, 18(12):2135-2143, 2015.

[12] C. Kim, Y. Gui, and C. C. Chung. Coordinated wind power plant control for frequency support under wake effects. In 2015 IEEE Power Energy Society General Meeting, pages 1-5, 2015.

[13] E. Bossanyi and T. Jorge. Optimisation of wind plant sector management for energy and loads. In 2016 European Control Conference (ECC), pages 922-927, 2016.

[14] Jacob Aho, Lucy Pao, and Paul Fleming. An Active Power Control System for Wind Turbines Capable of Primary and Secondary Frequency Control for Supporting Grid Reliability. 2013.

[15] C. R. Shapiro, J. Meyers, C. Meneveau, and D. F. Gayme. Coordinated pitch and torque control of wind farms for power tracking. In 2018 Annual American Control Conference (ACC), pages 688-694, 2018.

[16] J.M. Jonkman, S. Butterfield, W. Musial, and G. Scott. Definition of a 5mw reference wind turbine for offshore system development. National Renewable Energy Laboratory (NREL), 2009. 
[17] Matthew J. Churchfield, Sang Lee, Patrick J. Moriarty, Yujia Hao, Matthew A. Lackner, Rebecca Barthelmie, Julie K. Lundquist, and Gregory Oxley. A Comparison of the Dynamic Wake Meandering Model, LargeEddy Simulation, and Field Data at the Egmond aan Zee Offshore Wind Plant. 2015.

[18] J Jonkman, Paula Doubrawa, N Hamilton, Jennifer Annoni, and Paul Fleming. Validation of fast.farm against large-eddy simulations. Journal of Physics: Conference Series, 1037:062005, 2018.

[19] Paula Doubrawa, Jennifer R. Annoni, and Jason M. Jonkman. Optimization-Based Calibration of FAST.Farm Parameters against Large-Eddy Simulations. 2018.

[20] D Astrain Juangarcia, I Eguinoa, and T Knudsen. Derating a single wind farm turbine for reducing its wake and fatigue. Journal of Physics: Conference Series, 1037, 2018.

[21] J.M. Jonkman, G.J. Hayman, B.J. Jonkman, and R.R. Damiani. AeroDyn v15 User's Guide and Theory Manual. National Renewable Energy Laboratory (NREL), 2015. 